Revista Eureka sobre Enseñanza y Divulgación de las Ciencias

ISSN: 1697-011X

revista.eureka@uca.es

Universidad de Cádiz

España

\title{
Pedagogías del Riesgo: alfabetización científica en tiempos de pandemia
}

\author{
Valladares, Liliana \\ Pedagogías del Riesgo: alfabetización científica en tiempos de pandemia \\ Revista Eureka sobre Enseñanza y Divulgación de las Ciencias, vol. 19, núm. 1, 2022 \\ Universidad de Cádiz, España \\ Disponible en: https://www.redalyc.org/articulo.oa?id=92068491002 \\ DOI: https://doi.org/10.25267/Rev_Eureka_ensen_divulg_cienc.2022.v19.i1.1301
}




\section{Pedagogías del Riesgo: alfabetización científica en tiempos de pandemia}

Risk Pedagogies: scientific literacy in pandemic times

Liliana Valladares

Facultad de Filosofia y Letras, Universidad Nacional

Autónoma de México. Ciudad de México, México

lvalladares@comunidad.unam.mx

(DD https://orcid.org/0000-0001-9616-7021
DOI: https://doi.org/10.25267/

Rev_Eureka_ensen_divulg_cienc.2022.v19.i1.1301 Redalyc: https://www.redalyc.org/articulo.oa? $\mathrm{id}=92068491002$

Recepción: 21 Mayo 2020

Revisado: 03 Febrero 2021

Aprobación: 18 Julio 2021

\section{Resumen:}

En este artículo se describe en qué consiste la competencia en riesgo y se revisa su inclusión en la educación de ciencias. Se identifican y analizan las principales propuestas teórico-metodológicas, reportadas en la literatura especializada, para el tratamiento pedagógico del riesgo, y se discute su relación con los principales paradigmas teóricos que definen el riesgo. Se argumenta la pertinencia de incorporar el esquema SSI a las principales propuestas para la enseñanza del riesgo, y se plantea un metamodelo didáctico para facilitar la implementación de una pedagogía del riesgo en el aula de ciencias.

Palabras ClaVe: Riesgo, Educación científica, Competencia en riesgo, Pedagogía del riesgo.

\section{Abstract:}

This article describes the risk competence and its inclusion in science education. The main theoretical-methodological proposals, reported in the specialized literature for the pedagogical treatment of risk, are identified and analyzed, and their relationship with the main theoretical paradigms that define risk is discussed. The relevance of incorporating the SSI scheme into the main proposals for risk teaching is argued, and a didactic metamodel is proposed to facilitate the implementation of a risk pedagogy at the science school.

KEYWORDS: Risk, Science education, Risk competence, Risk pegagogy.

\section{INTRODUCCIÓN}

La sociedad actual se caracteriza por la incertidumbre, una falta de certeza que hace imposible conocer la probabilidad de que un cierto evento suceda (Mack y Khare 2016), y también en ella están presentes peligros, amenazas, y riesgos. Frecuentemente vivimos defendiéndonos de potenciales daños y muchas de nuestras prácticas cotidianas (lavarse las manos, abrocharse los cinturones de seguridad, cerrar las puertas, entre otras) buscan evitar los riesgos.

El riesgo, como probabilidad de que suceda un daño (Mack y Khare 2016), es parte de nuestras vidas y se presenta de diversas formas; puede ser voluntario o impuesto; personal o público; local o global: "Ya sea visible, invisible, tecnológico o natural, calculable o incalculable, siempre que los seres humanos participen en una acción propositiva, orientada al futuro, encuentran la posibilidad de un accidente y tratan de controlar esa posibilidad" (Bialostok 2015, p. 562).

Con frecuencia, el riesgo está asociado al desarrollo de la ciencia y la tecnología; acompaña, por ejemplo, a los nuevos artefactos tecnológicos o procedimientos médicos, bio o nanotecnológicos. Otras veces está asociado a un devenir aparentemente natural, que invariablemente tiene un componente antropogénico como en el cambio climático o la aparición de nuevas enfermedades, como ha sido el caso de la pandemia producida por el virus SARS-CoV-2, cuyo primer brote fue registrado en diciembre de 2019 en Wuhan (China). 
El caso de esta pandemia ha sido de enorme relevancia para reencontrarnos con la presencia ubicua del riesgo en nuestras vidas. Las consecuencias devastadoras de esta enfermedad infecciosa están siendo y serán significativas en ámbitos como la salud, la economía, la educación y la sociedad.

De acuerdo con el Reporte de Riesgos Globales 2019 (FEM 2019), la presentación de una pandemia no estaba incluida dentro de los 5 principales riesgos globales; se le estimaba como un riesgo de muy baja probabilidad, aunque de alto impacto. El 30 de enero de 2020 la epidemia de COVID 19 fue declarada por la Organización Mundial de la Salud una emergencia de salud pública de preocupación internacional y fue caracterizada como una pandemia, con riesgos sanitarios, económicos y socioculturales.

El enorme impacto de esta se comenzó a vislumbrar cuando, a fines de febrero de 2020, y con la rápida propagación de la COVID-19, los países afectados tomaron como principal medida el distanciamiento social, lo cual implicó el cierre masivo de comercios y de escuelas y la consecuente profundización de las desigualdades sociales, económicas y educativas.

Del total mundial de la población de estudiantes inscritos en la educación, la UNESCO calcula que, durante abril de 2020, casi 1,600 millones de alumnos estaban siendo afectados (más del 70\% de la matrícula), en alrededor de 192 países, por el cierre de las escuelas (Martínez y Diez 2020).

El reconocimiento creciente de que los riesgos y la incertidumbre son una parte inevitable y constitutiva de las sociedades contemporáneas nos exige preguntarnos acerca de cuál es la relevancia de abordar el tema del riesgo en el ámbito escolar, qué es y cómo se desarrolla la "competencia en riesgo", y cómo desde la educación en ciencias podríamos implementar una alfabetización en riesgo que nos haga capaces de transitar en este mundo cada vez más incierto y riesgoso (Beck, 1992).

Para dar respuesta a estas cuestiones, en el presente artículo se describe en qué consiste la competencia en riesgo, y se revisa su inclusión en la clase de ciencias. A la luz de los debates conceptuales sobre el riesgo, se analizan las principales propuestas teórico-metodológicas para el tratamiento pedagógico del riesgo reportadas en la literatura especializada y, recuperando las cualidades más relevantes de estas propuestas, se plantea un metamodelo didáctico que facilite la implementación de una pedagogía del riesgo en el aula de ciencias.

\section{Conceptualizaciones DeL RiEsgo}

Aunque la noción de riesgo surgió a mediados del siglo XVI, vinculada al comercio marítimo y la seguridad de los barcos, como campo de investigación se ha desarrollado fructíferamente desde hace tres décadas (Bialostok 2015). En su inicio fue un campo dominado por las ciencias naturales, con orientación principalmente hacia la identificación y la medición, que concebía al riesgo como la frecuencia relativa de un evento medido en un período de tiempo pasado o como la cuantificación de la relación entre un agente de riesgo potencial y el daño físico observado; posteriormente, las ciencias sociales complejizaron esta definición, cambiando el foco de atención de la identificación o evaluación hacia los ámbitos de la gestión, percepción y comunicación del riesgo (Christensen 2009, Bialostok 2015).

Son múltiples y multívocas las definiciones sobre el riesgo (Bialostok 2015) y esto ha derivado en una ambigüedad en el uso término que con frecuencia se confunde con el peligro, la amenaza, el daño, la vulnerabilidad o la incertidumbre (Hansen y Hammann 2017).

La mayoría de las definiciones de riesgo suponen que los eventos futuros no son controlados ni predeterminados y distinguen entre la posibilidad y la realidad de que ocurran (Renn 1992, Rodríguez 2007). La Tabla 1 sintetiza algunas de las tipologías para definir el riesgo. En esta tabla se puede apreciar que predominan dos grandes paradigmas sobre el riesgo: el técnico, preponderante en las ciencias naturales, que lo define como entidad objetiva, que se puede definir y calcular; y el subjetivo, más común en las ciencias sociales, que lo examinan como una construcción social que se inscribe en prácticas institucionales que diversifican su percepción (Jassanoff 1999, Rodríguez 2007 y Bialostok 2015). 
TABLA 1

Tipologías y concepciones sobre el riesgo

\begin{tabular}{|c|c|c|}
\hline Autor (es) & Categorías del riesgo & Concepciones del riesgo \\
\hline \multirow{8}{*}{ Renn (1992) } & 1) Aproximación actuarial & \multirow{8}{*}{$\begin{array}{l}\text { Riesgo como resultado de la relación entre la posibilidad de } \\
\text { ocurrencia (de un acontecimiento); sus resultados o conse- } \\
\text { cuencias no deseadas (efectos perversos o colaterales); y el } \\
\text { estado de realidad (cómo están las cosas). } \\
\text { Difieren por sus métodos de evaluación. } \\
\text { 1-4: ven al riesgo como propiedad objetiva de un evento o } \\
\text { de una actividad, medida como la probabilidad de que su- } \\
\text { ceda y la magnitud del daño posible. } \\
\text { 5-8: ven al riesgo como construcción social subjetiva, per- } \\
\text { cibida y negociada. }\end{array}$} \\
\hline & $\begin{array}{l}\text { 2) Aproximación epidemiológi- } \\
\text { ca y toxicológica }\end{array}$ & \\
\hline & $\begin{array}{l}\text { 3) Aproximación técnica o in- } \\
\text { genieril }\end{array}$ & \\
\hline & 4) Aproximación económica & \\
\hline & 5) Aproximación psicológica & \\
\hline & 6) Teorías sociales del riesgo & \\
\hline & 7) Teoría cultural del riesgo & \\
\hline & 8) Aproximación jurídica & \\
\hline \multirow{3}{*}{$\begin{array}{l}\text { Jasanoff } \\
(1999)\end{array}$} & 1) Riesgo técnico & $\begin{array}{l}\text { Riesgo como subproducto tangible de un proceso natural o } \\
\text { social, que puede mapearse y medirse por expertos, y con- } \\
\text { trolarse y limitarse. }\end{array}$ \\
\hline & 2) Riesgo construido & $\begin{array}{l}\text { Riesgo como constructo determinado y permeado por la } \\
\text { sociedad, la historia, la política y la cultura, cuya regulación } \\
\text { y gobierno requiere de la negociación entre diferentes per- } \\
\text { cepciones, no reducidas al conocimiento técnico. }\end{array}$ \\
\hline & $\begin{array}{l}\text { 3) Riesgo como dispositivo de } \\
\text { poder }\end{array}$ & $\begin{array}{l}\text { Riesgo foucaultiano, mediador entre el conocimiento y el } \\
\text { poder, que empodera a algunos grupos sociales y excluye a } \\
\text { otros. }\end{array}$ \\
\hline \multirow{3}{*}{$\begin{array}{l}\text { Bialostok y } \\
\text { Whitman } \\
\text { (2012) y Bia- } \\
\text { lostok } \\
\text { (2015) }\end{array}$} & 1) Sociedad del riesgo & $\begin{array}{l}\text { Riesgo como rasgo social inevitable y característico de la } \\
\text { sociedad actual (segunda modernidad), vinculado a la refle- } \\
\text { xividad, y que en lo global se produce y distribuye diferen- } \\
\text { cialmente. }\end{array}$ \\
\hline & 2) Enfoque cultural & $\begin{array}{l}\text { Riesgo como invención social producto de la intersección } \\
\text { de valores, creencias y percepciones; culturalmente cons- } \\
\text { truido, pues lo que una sociedad considera un riesgo signi- } \\
\text { ficativo, en otra puede pasar desapercibido. }\end{array}$ \\
\hline & $\begin{array}{l}\text { 3) Enfoque de gubernamentali- } \\
\text { dad }\end{array}$ & $\begin{array}{l}\text { Riesgo como técnica del gobierno y forma de poder disci- } \\
\text { plinario que trabaja a través y sobre el individuo. }\end{array}$ \\
\hline \multirow{3}{*}{$\begin{array}{l}\text { Rodríguez } \\
\quad(2007\end{array}$} & 1) Teorías técnicas del riesgo & $\begin{array}{l}\text { Anticipan, identifican y cuantifican las consecuencias nega- } \\
\text { tivas potenciales y hacen una radiografía de escenarios fu- } \\
\text { turos posibles a partir del estudio de la realidad. }\end{array}$ \\
\hline & $\begin{array}{l}\text { 2) Teorías de la representación } \\
\text { pública del riesgo }\end{array}$ & $\begin{array}{l}\text { Indagan las razones que subyacen a la resistencia pública } \\
\text { hacia la ciencia y la tecnología y sus consecuencias. }\end{array}$ \\
\hline & 3) Teorías sociales del riesgo & $\begin{array}{l}\text { Riesgo como experiencia social que permite comprender a } \\
\text { las sociedades contemporáneas. }\end{array}$ \\
\hline
\end{tabular}

Los dos paradigmas (objetivo y subjetivo) son necesarios para comprender el riesgo, pues este tiene una constitución heterogénea (Rodríguez (2007) que lo define como un fenómeno poliédrico constituido por elementos epistemológicos, técnicos, políticos, ecológicos, públicos, económicos, en donde la subjetividad de las percepciones de riesgo se extiende también a los científicos e ingenieros, cuyas estimaciones cuantitativas del riesgo están igualmente influidas por factores psicológicos, sociales, institucionales y culturales (Christensen 2009).

Dado que la pandemia puso de relieve los riesgos constantes que enfrentamos día con día y la importancia de saber reconocerlos y gestionarlos, en este trabajo se explora qué tanto han permeado en el ámbito escolar estas discusiones teóricas para conceptualizar el riesgo, y cuáles propuestas pedagógicas se han planteado para su enseñanza. 


\section{Metodología}

Para conocer si están presentes y en qué forma se traducen las concepciones teóricas del riesgo en el ámbito escolar, se realizó un análisis documental sobre la literatura especializada en el tratamiento del riesgo en la educación, y particularmente en la educación científica, en los últimos cincuenta años.

Las bases de datos consultadas fueron ERIC, Springer, Taylor \& Francis, Wiley, Sage y BiDi-UNAM y los criterios de selección de la literatura fueron: a) que el escrito incluyera una reflexión/propuesta pedagógica sobre el riesgo; b) que el tema del riesgo estuviera vinculado a la educación en ciencias. Se realizó un análisis de contenido de los trabajos encontrados en función de cuatro preguntas de investigación: 1) ¿cómo ha sido abordada la enseñanza del riesgo en el aula de ciencias?, 2) ¿en qué consiste la llamada competencia en riesgo?, 3) ¿cómo se establece la relación entre las múltiples concepciones teóricas del riesgo y su tratamiento en el aula? 4) ¿qué objetivos y dimensiones caracterizan a una pedagogía del riesgo?

A partir de los resultados obtenidos se sistematizaron y analizaron los componentes mínimos que definen a la competencia en riesgo. Se caracterizaron las propuestas pedagógicas para el tratamiento educativo del riesgo, en función de su vínculo con los avances teóricos para definir el riesgo, identificándose dos propuestas pedagógicas que se clasificaron como de alta relevancia para los fines de este escrito, al ser las únicas que explicitaron una relación entre la forma en cómo se conceptualiza teóricamente el riesgo y el tratamiento pedagógico sugerido para este tema dentro de la alfabetización en ciencias.

Como parte del análisis de dichas propuestas, se identificaron fortalezas y debilidades para su implementación en el aula de ciencias, y a partir de ello se construyó un metamodelo didáctico con orientaciones y pistas pedagógicas que ejemplifican cómo se podría facilitar al profesorado la enseñanza del riesgo. Este metamodelo, pendiente de llevarse a la práctica, se compromete con una concepción heterogénea del riesgo, y propone un objetivo, principios y dimensiones instruccionales básicas para lograr que desde la alfabetización en ciencias se propicie el desarrollo de una pedagogía del riesgo.

\section{Resultado 1: LA TRAYECTORIA DEL RIESgo EN LA EDUCACión EN CIENCIAS}

La educación en ciencias se ha convertido en un campo relevante para abordar el tema del riesgo, pues el avance científico no solamente se ha acompañado de riesgos, sino que es la ciencia la que, a través de sus órganos sensoriales -como pueden ser las teorías, los experimentos, los instrumentos de medición, entre otros-, vuelve visibles y medibles estos riesgos en su mayoría imperceptibles (Christensen 2009).

Los trabajos de Ryder (2001), Millar (2006), Kolstø (2006) entre otros, han dado cuenta de la relevancia del riesgo como parte de la alfabetización científica, sobre todo aquella desarrollada bajo el paradigma de Ciencia, Tecnología y Sociedad (CTS), en la medida en que el riesgo contribuye a la comprensión de los aspectos sociales y epistemológicos de la ciencia (Schenk, Hamza, Enghag, Lundegård Engh, Arvanitis Haglund y Wojcik 2019).

En la educación CTS, particularmente en su vertiente conocida como SSI, el riesgo se incluye como una de las características de estas controversias (Pedretti y Nazir 2011, 2016). No obstante, como un contenido especifico e independiente, el riesgo apenas se aborda en algunos programas de enseñanza de las ciencias (Christensen 2009), a pesar de que en las décadas de los ochenta y noventa, autores como Howes (1975), Eijkelhof (1986), y Ravetz (1997), abogaron por su inclusión en la educación científica, y en el caso de Riechard (1993), como un unificador de la educación ambiental.

De acuerdo con Ravetz (1997), en la educación científica ha habido una exclusión sistemática de la incertidumbre, la ignorancia y el riesgo como aspectos característicos de la ciencia. Para este autor, la educación en ciencias debería promover, junto con las preguntas quéy cómo, la pregunta ¿quétalsi? (what if?), pues esta genera una conciencia sobre las incertidumbres de la ciencia y transforma la idea equivocada de que esta es un cuerpo de conocimientos coherente, objetivo, a-problemático, carente de riesgos e incertidumbres. 
La pregunta what if? expresa un espíritu científico de creatividad e invención y está englobada en cualquier análisis del riesgo, no obstante, está ausente en casi toda práctica educativa de la ciencia (Ravetz 1997).

Si bien ha habido un creciente compromiso por incluir el riesgo en los currículos de ciencia en general, y en esto han avanzado países como Reino Unido, Estados Unidos, Canadá y Australia (Hansen y Hamman 2017), todavía es poca la investigación sobre la comprensión de los estudiantes acerca del riesgo o el papel que este desempeña en la toma de decisiones sobre problemas sociocientíficos contemporáneos en las aulas de ciencias (Christensen 2009). El concepto de riesgo es controvertido y rara vez se ha tratado con profundidad en la literatura de educación científica y, aun cuando casi la mitad de los estudios analizados se centran específicamente en el riesgo como contenido didáctico y proporcionan una definición del concepto, sólo los trabajos de Christensen (2009), Hansen y Hammann (2017) y Schenk et al (2019), discuten la correlación entre la conceptualización teórica del riesgo que se adopta en el currículo y su tratamiento didáctico, siendo los últimos dos trabajos los únicos que incluyen una propuesta pedagógica sólida para la enseñanza del riesgo en la clase de ciencias.

\section{Resultado 2: El SignifiCAdo DE LA COMPETENCIA EN RIESGO}

La competencia en riesgo puede comprenderse como un componente central de la alfabetización científica, y a partir del análisis documental puede inferirse que su desarrollo y fortalecimiento depende en gran medida de conocer dos aspectos: 1) cómo se conceptualiza el riesgo (sus marcos teóricos); 2) cómo estas definiciones determinan cómo ha de enfrentarse el riesgo y cómo puede trabajarse pedagógicamente en el aula de ciencias.

De acuerdo con Nikiforidou (2017), aprender a asumir y gestionar el riesgo a nivel individual, está interconectado con diferentes aspectos del desarrollo cognitivo, social, emocional y biológico. La competencia en riesgo, por consiguiente, está permeada por diferencias personales (edad, género y experiencias previas) y factores socioculturales, asociados al contexto y a las interacciones y prácticas de socialización (Nikiforidou 2017).

Según esta autora, el riesgo debe abordarse desde la educación inicial (primera infancia), pues serviría como un vehículo para alfabetizar a los niños en riesgo, lo que significa desarrollar su capacidad de juzgar y emprender riesgos y enfrentar la incertidumbre y la inestabilidad: "Si los niños no desarrollan la capacidad para razonar y asumir las consecuencias de sus decisiones, no podrán hacer frente a los riesgos y peligros, oportunidades y desafíos, no solo en la actualidad, sino también en su futuro" (Nikiforidou 2017, p. 323).

Siguiendo a Nikiforidou (2017), aprender a analizar el riesgo y confrontarlo (esto incluye, la identificación, evaluación, gestión y comunicación de riesgos), implica dos procesos no lineales y entretejidos: a) el pensamiento y comprensión objetivos y subjetivos, es decir, la conciencia y percepción de la situación de riesgo, y b) el compromiso o evitación del riesgo, esto es, la acción y comportamiento que se asume ante la situación riesgosa. Ambos procesos configuran a la competencia en riesgo.

El primer proceso (pensar y comprender el riesgo) puede, a su vez, descomponerse en dos elementos subyacentes: al) los de tipo cognitivo, que permiten entender objetivamente el riesgo, a través de cálculos y especulaciones relacionadas con el conocimiento estadístico y matemático, con el pensamiento probabilístico, el conocimiento contextual y las capacidades críticas necesarias para cuantificar medir y anticipar el riesgo; y a2) los de tipo disposicional, que permiten construir interpretaciones personales del riesgo, y que están vinculadas con aspectos no cognitivos como las creencias, emociones y actitudes subjetivas de cada individuo. Ambos elementos (cognitivos/objetivos y disposicionales/subjetivos) orientarán el segundo proceso, esto es, la toma de decisiones y acciones que se emprenden en torno al riesgo.

Aunque la preparación para el riesgo abarca un aprendizaje a lo largo de toda la vida, la competencia en riesgo se puede desarrollar a partir de la edad de 4 años, cuando los niños son capaces de comprender y racionalizar situaciones de seguridad/no seguridad y distinguir entre riesgos aceptables e inaceptables, y entre sentirse seguro y estar a salvo. 
La competencia en riesgo puede definirse así como la habilidad y actitud para toda la vida que forma agentes educados para identificar, comunicar, gestionar y evaluar el riesgo en diversas situaciones, tanto a nivel cognitivo como disposicional o de comportamiento.

Alfabetizarse en riesgo, según Nikiforidou (2017), requiere la enseñanza de una triada de elementos interconectados por un enfoque curricular transversal, que no se limite solo a la estadística o las matemáticas, como sucede usualmente, sino que articule otras áreas humanísticas como la educación cívica y ética. La triada abarca la capacidad de usar representaciones gráficas (y que permite entender y comunicar ideas, relacionar información a través de mapas, diagramas, gráficas, símbolos, que pueden ser usados para fomentar el pensamiento estadístico necesario para describir, organizar, representar e interpretar datos para hacer inferencias sobre el mundo); el razonamiento probabilístico (que permite pensar, explicar y procesar posibilidades y probabilidades, eventos futuros, caminos alternativos, elecciones inciertas, opciones posibles y resultados probables), y; el desarrollo de una cultura del riesgo en el aula, que implica adoptar actitudes y comportamientos hacia la toma de riesgos, que superen su percepción mayormente negativa, para ser comprendidos positivamente como posibilidad, cambio, autonomía, novedad y creatividad (Nikiforidou 2017).

Hansen y Hammann (2017) coinciden con Nikiforidou en proponer que la percepción, identificación, evaluación, gestión y decisión sobre el riesgo, es un proceso complejo que convoca una amplia gama de habilidades para tratarlo en un ambiente real. Para estos autores la competencia en riesgo es compuesta y multidimensional y dado que el riesgo está siempre presente en la ciencia, la enseñanza de la ciencia no debería omitir su estudio.

\section{Resultado 3: Tratamiento Pedagógico del Riesgo en la enseñanza de las Ciencias}

Del análisis documental sobre el tratamiento educativo del riesgo, destacan dos propuestas pedagógicas vinculadas: a) la propuesta pedagógica de Hansen y Hammann (2017), de carácter reconciliador, porque subraya la necesidad de primero comprender cómo se conceptualiza el riesgo, combinando sus paradigmas, para luego poder enseñarlo como un tópico más dentro de la enseñanza de la ciencia; y b) la propuesta pedagógica de Schenk et al (2019), de carácter poliédrico, en cuanto a que enfatiza los aspectos procedimentales y actitudinales del riesgo, enmarcándolo como un atributo de toda actividad humana, que también atraviesa y es intrínseco al quehacer de la ciencia.

Ambas propuestas son relevantes porque reconocen que según la forma en cómo se conceptualiza el riesgo, este será enseñado. Christensen (2009) fue de los primeros en advertir la importancia que tiene para un educador en ciencias primero comprender cómo se define y concibe teóricamente el riesgo, pues en función de ello estará condicionada y pueden determinarse los mejores caminos para su enseñanza.

\section{Entre lo objetivo y lo subjetivo: la propuesta pedagógica reconciliadora de Hansen y Hammann}

Desarrollada por Hansen y Hammann (2017), esta aproximación pedagógica reconoce la constitución heterogénea del riesgo y reconcilia los dos grandes paradigmas conceptuales: el paradigma objetivo o técnico y el constructivista o subjetivo.

Hansen y Hammann (2017) asocian a cada paradigma conceptual sobre el riesgo un conjunto de características para entender por un lado, cómo se define el riesgo en dicho paradigma, y por otro lado, cuáles son las implicaciones que cada paradigma tiene en la educación científica.

La Tabla 2 sintetiza esta relación y destaca la relevancia de reconciliar ambos paradigmas y configurar un abordaje didáctico integrador del riesgo, que no lo reduzca a sus dimensiones técnicas, exigiendo a los 
estudiantes el ajuste cognitivo necesario para sustituir sus percepciones sociales y culturales del riesgo, por las estimaciones técnicas del mismo. El riesgo se constituye de ambos paradigmas y su enseñanza debe siempre considerarlo.

TABLA 2

Paradigmas conceptuales sobre el riesgo en la educación científica

\begin{tabular}{|c|l|}
\hline Paradigma & \multicolumn{1}{c|}{ Implicaciones educativas } \\
\hline $\begin{array}{c}\text { Positivista/objetivo } \\
\text { (riesgo técnico) }\end{array}$ & $\begin{array}{l}\text { Énfasis en los conocimientos de estadística y matemáticas; teoría de la probabi- } \\
\text { lidad; y conocimiento científico de tópicos de ciencias naturales. } \\
\text { expertos de riesgo, para gestionarlos y evaluar la probabilidad de su impacto. } \\
\text { Medios de comunicación e informativos se consideran simplificadores de los } \\
\text { estudios científicos. }\end{array}$ \\
\hline $\begin{array}{c}\text { Constructivista/subjeti- } \\
\text { vo (riesgo percibido) }\end{array}$ & $\begin{array}{l}\text { La alfabetización no se reduce a ajustar y corregir cognitivamente la percepción } \\
\text { de riesgo de los estudiantes para conducirla hacia el riesgo técnico. } \\
\text { de los problemas de riesgo. } \\
\text { Reflexión sobre el riesgo como socialmente percibido y definitorio de las activi- } \\
\text { dades científico-tecnológicas. }\end{array}$ \\
\hline
\end{tabular}

La propuesta pedagógica de Hansen y Hammann (2017), condensada en la Tabla 3, consiste reconciliar ambos paradigmas.

TABLA 3

Propuesta pedagógica de Hansen y Hammann

\begin{tabular}{|c|c|}
\hline $\begin{array}{l}\text { Constitución heterogé- } \\
\text { nea del riesgo } \\
\text { (comprende ambos pa- } \\
\text { radigmas) }\end{array}$ & $\begin{array}{l}\text { Desarrollo integral de las habilidades de los paradigmas técnico y constructivista. } \\
\text { Se estudia a la ciencia en contexto como una práctica social incierta, embebida en } \\
\text { sistemas de valores socioculturales. } \\
\text { Enseñanza-aprendizaje de } 3 \text { componentes: } \\
\text { 1) Conocimiento cientifico y de estadística/probabilidad asociado al riesgo bajo estudio, y } \\
\text { contenido en los análisis de riesgo elaborados por expertos. } \\
\text { 2) Conocimiento sobre la ciencia a la que ubica en una red social más amplia, destacan- } \\
\text { do su incertidumbre, sus limitaciones, intereses en juego, juicios de valor y facto- } \\
\text { res sociales. } \\
\text { 3) Habilidades para la evaluación de riesgos asociadas a la identificación y evaluación } \\
\text { de riesgos (y beneficios); de resultados alternativos; el análisis de riesgo-beneficio } \\
\text { y la ponderación; la argumentación y la toma de decisiones. }\end{array}$ \\
\hline
\end{tabular}


En esta propuesta al mismo que se propicia un conocimiento técnico sobre el riesgo, se promueve también un conocimiento de su carácter social y construido, facilitando su combinación para que los estudiantes evalúen, sopesen y tomen, de manera individual o colectiva, una decisión argumentada sobre los riesgos en cuestión (Hansen y Hammann 2017).

\section{La actividad en el centro: la propuesta pedagógica poliédrica de Schenk et al}

Desarrollada por Schenk et al (2019), esta segunda propuesta para la enseñanza del riesgo es resultado del análisis de definiciones del riesgo y de su uso en los campos de ingeniería, lingüística y filosofía.

Schenk et al (2019) identificaron 7 componentes clave para orientar la planificación, ejecución y evaluación de actividades de enseñanza que impliquen explícita o implícitamente problemas de riesgo. A diferencia de la propuesta reconciliadora, esta va más allá de reconocer la naturaleza compuesta (subjetiva/ objetiva) del riesgo, para ubicarlo como parte constitutiva de las actividades humanas. La noción de actividad es clave para enseñar sobre el riesgo, porque a esta puede asociarse un conjunto de consecuencias; y las consecuencias se acompañan siempre de atributos como la incertidumbre, la probabilidad y la gravedad.

De este modo, Schenk et al (2019), proponen 4 componentes nucleares o internos del riesgo: 1) consecuencia, 2) probabilidad, 3) incertidumbre y 4) severidad o gravedad. A estos se añaden 3 componentes externos del riesgo que permiten contextualizar su enseñanza: 5) actividad, 6) conocimiento y 7) valores. Al ser resultado de una toma de decisiones, el riesgo conlleva el componente del conocimiento que se usa para decidir las acciones a emprender en torno a una actividad, y esta decisión se toma en función de un conjunto de valores. En la Tabla 4 se definen estos componentes.

TABLA 4

Componentes nucleares y externos del riesgo

\begin{tabular}{|c|l|}
\hline Componente nuclear & \multicolumn{1}{c|}{ Definición } \\
\hline Consecuencia & Evento, resultado, o pérdida asociados a una actividad \\
\hline Probabilidad & Medio para operacionalizar cuantitativamente la incertidumbre \\
\hline Incertidumbre & $\begin{array}{l}\text { i) Incertidumbre aleatoria (causada por el azar intrínseco a cualquier } \\
\text { evento). } \\
\text { ii) Incertidumbre epistémica (causada por falta de conocimiento } \\
\text {-por lo que teóricamente puede reducirse) }\end{array}$ \\
\hline Severidad/gravedad & Naturaleza o grado de adversidad de la consecuencia \\
\hline Actividad & Conjunto de acciones emprendidas por los seres humanos \\
\hline Conocimiento & Información valorada que se usa para decidir y actuar \\
\hline Valores & $\begin{array}{l}\text { Creencias e interpretaciones sobre lo que resulta valioso para un } \\
\text { grupo social en un momento dado }\end{array}$ \\
\hline
\end{tabular}

La propuesta de Schenk et al (2019) subraya la relevancia de que en el contexto educativo se fomente una aproximación al riesgo multifacética y compuesta, como lo ilustra la Figura 1, por un núcleo de componentes internos (consecuencia, incertidumbre, probabilidad y gravedad), enmarcados por otros contextuales que son producto de las decisiones tomadas en las actividades humanas (actividad, conocimiento y valores). 


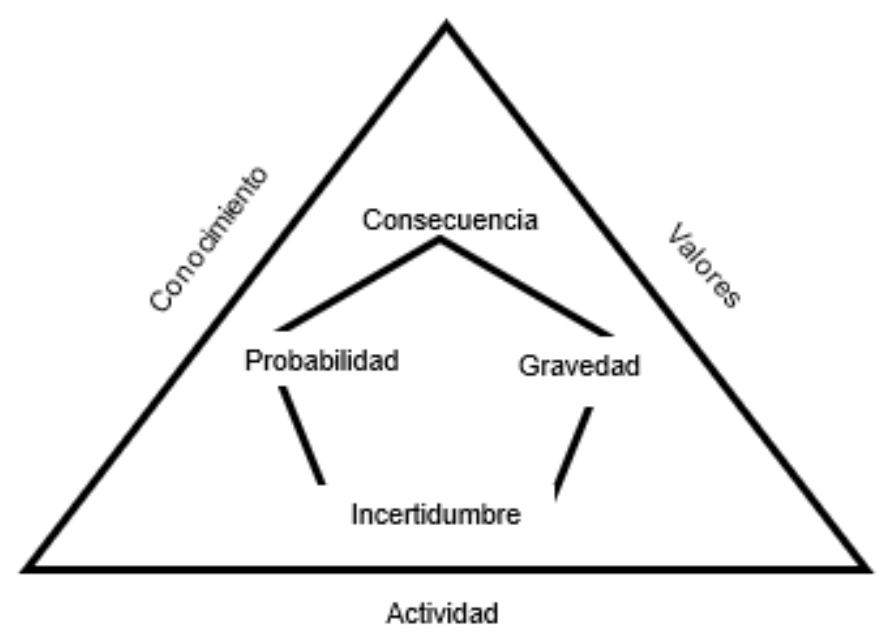

FIGURA 1

Componentes nucleares y contextuales del riesgo. Traducido de Schenk et al (2019, p. 1278).

De acuerdo con la Figura 1, la actividad se convierte en el punto de anclaje para identificar y abordar el riesgo en el aula. Mientras que con el polígono interno los profesores pueden enseñar sobre el riesgo (teaching about risk), -sus probabilidades, incertidumbres, daños, consecuencias asociadas-, con el triángulo externo los profesores pueden enseñar a través del riesgo (teaching through risk), esto es, indagando acerca del riesgo en las actividades humanas.

En su implementación práctica, los profesores de ciencias pueden elegir trabajar con todos o con solo un conjunto de los componentes y explicar las relaciones entre ellos. Por ejemplo, se puede elegir del triángulo externo un contenido científico (conocimiento) y trabajar con los alumnos las actividades humanas asociadas a este, y del triángulo interno se pueden trabajar las consecuencias de estas actividades, con sus efectos, incertidumbres, probabilidades y gravedad (Schenk et al 2019).

\section{Propuesta didáctica emergente: Metamodelo pedagógico del riesgo}

Del análisis de estas dos propuestas se identificaron entre sus fortalezas el reconocer que la enseñanza del riesgo debe ser multifacética y contextualizada y que debe evitarse en el aula la reducción de los problemas de riesgo a una versión incompleta, constituida solo por números estadísticos y datos técnicos. Ambas propuestas ofrecen a los profesores orientaciones para abordar conceptualmente el riesgo, sin embargo, el componente identificado como ausente es un esquema instruccional coherente que facilite al profesorado pistas para su despliegue en el aula. Es decir, la enseñanza del riesgo no se da en aislado, como un tema independiente en la clase de ciencias, sino que el uso de estas dos propuestas requiere ser transversal a los diferentes contenidos de la ciencia escolar. Para lograr un aprendizaje experiencial, reflexivo y situado (Díaz, 2006) acerca del riesgo, hace falta un andamiaje sólido que permita aprovechar las cualidades de estas dos propuestas y que facilite ponerlas simultáneamente en acción, pues al mismo tiempo que el riesgo está intrínseco a toda actividad humana, como propone Schenk et al (2019), se constituye tanto de elementos subjetivos como objetivos, como proponen Hansen y Hammann (2017), pero también demanda del profesor ciertos atributos que le permitan construir un ambiente de la clase adecuado para desarrollar experiencias significativas en las que el estudiantado analice los riesgos en situaciones concretas y de relevancia para su vida real.

En este apartado se argumenta que el esquema instruccional que permitiría configurar el hábitat necesario para la implementación contextual de las propuestas revisadas es el de las llamadas cuestiones o problemas 
sociocientificos (SSI); este se ha mostrado potente en la enseñanza de las ciencias porque fomenta una educación-cientifica-en-contexto.

Las SSI son una vertiente de la educación CTS (Pedretti y Nazir 2011, 2016) que apareció en la literatura en 1986 (Sadler y Dawson 2012), y representó una posibilidad para lograr un cambio de la cultura convencional de la ciencia escolar (Sadler 2011). Mientras que la educación CTS parecía limitarse a enfatizar la interrelación entre ciencia-tecnología-sociedad, las SSI iban más allá e incluían las dimensiones éticas de la ciencia a la par del desarrollo moral y emocional de los estudiantes (Zeidler y Nichlos 2009).

El movimiento SSI es un marco conceptual, procedimental y tecnológico (Sadler y Donelly 2006) utilizado para guiar la planificación curricular y la pedagogía de la ciencia, así como la teoría, la investigación y la práctica en la educación científica (Zeidler 2016), que se distingue por utilizar tópicos científicos controversiales (Levinson 2006), para ayudar a los estudiantes a discutir, dialogar y debatir entre ellos, con el fin de proponer soluciones posibles a dichas controversias (Owens, Sadler y Zeidler 2017).

Las SSI se distinguen porque: a) son problemas controvertidos y abiertos (open-ended problems) (Díaz 2006), que requieren de un razonamiento basado en evidencia científica para informar las decisiones asociadas a estos problemas; b) usan de forma deliberada temas científicos en sus dimensiones sociales que exigen que los estudiantes participen en diálogo, discusión, debate y argumentación para su resolución; c) tienden a tener componentes éticos implícitos y explícitos, que requieren algún grado de razonamiento moral; d) estimulan la formación de la virtud, la conciencia y el carácter de los estudiantes como meta pedagógica de lago aliento, a través de un proceso continuo de normación, y pensamiento (auto)reflexivo, en el que los estudiantes evalúan y analizan sus propios razonamientos y acciones, cuestionando cómo pueden mejorarse (Zeidler 2016, Zeidler y Nichlos 2009).

Diversos estudios han mostrado que este esquema SSI tiene muchos resultados favorables para la educación en ciencias (Christensen 2009, Sadler y Dawson 2012, Lee, Yoo, Choi, Kim, Krajcik 2013, Zeidler 2016, Zeidler, Herman y Sadler 2019). Dadas sus enormes ventajas, la enseñanza del riesgo tecnocientífico puede beneficiarse sin duda del esquema SSI (Christensen 2009); Schenk et al 2019), pero ¿Cómo articularlo con las propuestas pedagógicas revisadas para el riesgo?

De acuerdo con Sadler (2011), el esquema SSI, en su sentido más amplio, comprende un marco instruccional flexible y adaptable a diversos objetivos de aprendizaje de la ciencia y del riesgo asociado a ella, a partir de considerar un conjunto de dimensiones entrelazadas.

Estas dimensiones abarcan, en primer lugar, el planteamiento de una cuestión sociocientífica en clase, es decir, un problema que evoque contenidos científicos y que sea socialmente relevante para un grupo escolar en un momento dado (por ejemplo, los riesgos de vacunarse o no contra la COVID-19); este anclaje a una SSI es crucial para mostrar al riesgo como siempre presente en cualquier contenido científico. En segundo lugar; el proporcionar a los estudiantes oportunidades para promover un tipo de razonamiento sociocientífico (SSR, por sus siglas en inglés), esto se refiere a prácticas de razonamiento asociado a la resolución de SSI y que incluyen capacidades de exploración, tránsito y articulación entre el escepticismo, la complejidad, la indagación y la toma de postura ante perspectivas múltiples (Zeidler y Sadler 2011, Zeidler, Herman y Sadler 2019). En el caso ejemplificado, se puede propiciar que el alumnado busque recopilar y analizar datos científicos sobre las consecuencias tanto de vacunarse como de no vacunarse, para posteriormente confrontar ideas y teorías científicas contrapuestas (por ejemplo, mediante un juego de roles simulando un debate público entre activistas provacunas y antivacunas) vía actividades dirigidas a participar en procesos de razonamiento, argumentación, toma de decisiones y de postura, y que den cabida a la explicitación y negociación de los aspectos sociales y éticos de la cuestión sociocientífica bajo estudio (i.e. la vacunación).

Pero el esquema SSI, no se queda solamente ahí, sino que también da pautas a los profesores para diseñar un ambiente de la clase de ciencias que potencie el aprendizaje, por ejemplo, propiciando expectativas de una alta participación estudiantil, de trabajo cooperativo, colaborativo e interactivo, que fomente el respeto mutuo y la confianza y seguridad. 
Esto implica que el profesor asuma un conjunto de prácticas que hagan posible esta forma de trabajo, entre ellas: Estar familiarizado con los problemas SSI bajo estudio; conocer el contenido de ciencias relacionado a este; ser consciente de las dimensiones sociales del SSI; ser honesto sobre las limitaciones del conocimiento; estar dispuesto a lidiar con las incertidumbres en el aula; estar dispuesto a posicionarse como un contribuyente más al proceso de conocimiento y no como autoridad única en el aula.

Si sumamos las virtudes del esquema SSI, con los aportes logrados por las propuestas pedagógicas de Hansen y Hammann (2017) y Schenk et al (2019), el riesgo puede entonces ser enseñado como un atributo compuesto, poliédrico y definitorio de la práctica científica que puede trabajarse y comprenderse dentro de alguna actividad humana explícitamente vinculada a una SSI. La cuestión sociocientífica seleccionada se convierte entonces en el eje para enseñar el riesgo; esta cuestión debe ser relevante y significativa para las experiencias de los estudiantes, pues sus consecuencias serán evaluadas y discutidas a la luz de los componentes nucleares del riesgo (su incertidumbre, probabilidad y gravedad), como de los componentes contextuales en que tiene lugar dicha actividad (valores prevalecientes y conocimiento científico y sobre la ciencia vinculados a ella). La Figura 2 sintetiza esta fusión de aproximaciones pedagógicas en un metamodelo para el análisis del riesgo en la clase de ciencias.

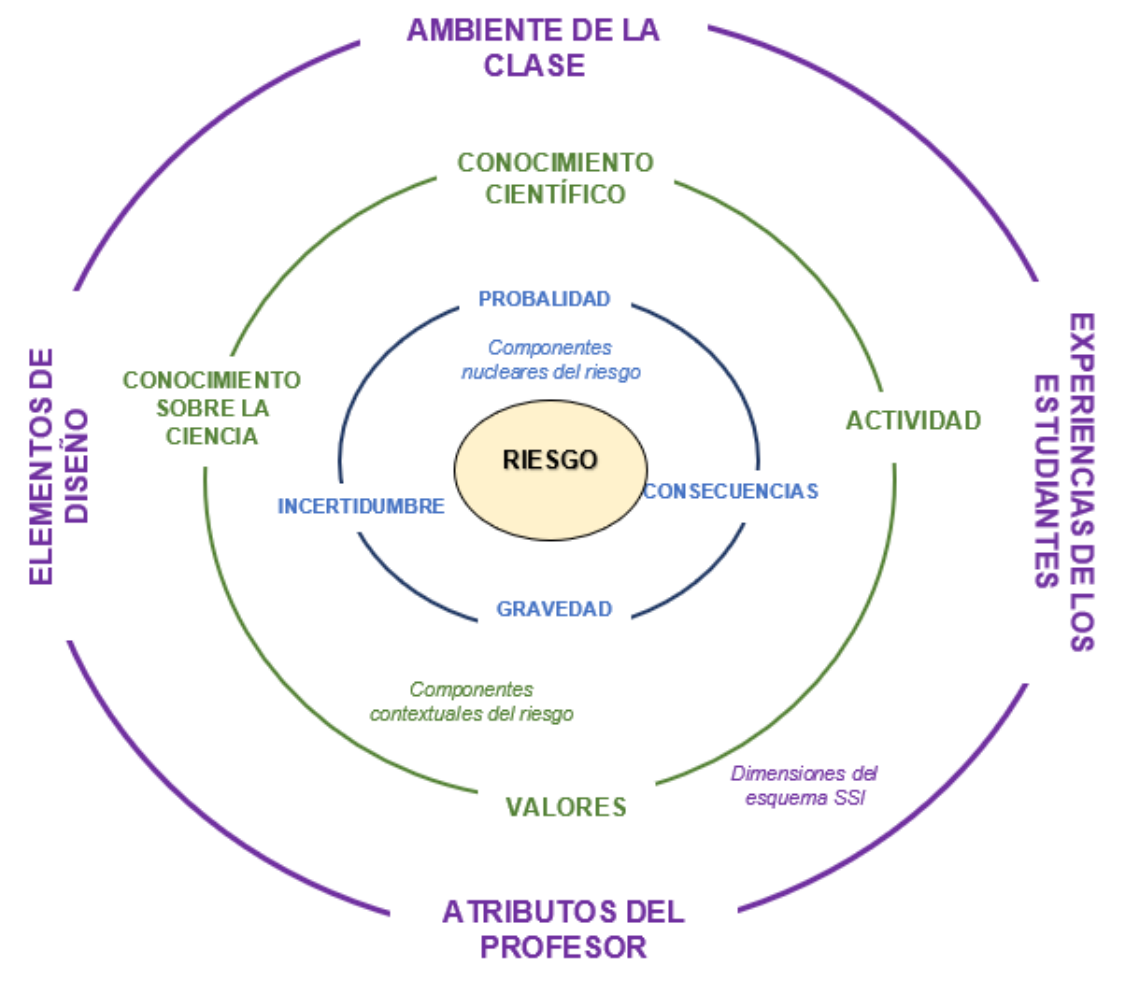

FIGURA 2

Metamodelo didáctico para una pedagogía del riesgo

Considerando el conjunto de elementos implicados en la Figura 2, una propuesta integral para la enseñanza del riesgo en la clase de ciencias requiere que el profesor: 1) introduzca a los estudiantes a una SSI, significativa social y personalmente; 2) evoque sus ideas previas sobre los riesgos asociados para discutirlas y analizar los aspectos nucleares y contextuales, objetivos y subjetivos de los riesgos implícitos en la SSI; 3) recopile las ideas clave de este análisis mediante dinámicas de clase propias del esquema SSI (por ejemplo, estudios de casos; desarrollo de proyectos colectivos y situados; debates públicos; reportajes sobre polémicas reales; encuestas sobre percepción pública de determinadas cuestiones polémicas relacionadas con la ciencia y la tecnología; análisis de contenidos en los medios de comunicación sobre el riesgo, entre otros) para que los estudiantes 
pongan en práctica procesos de razonamiento sociocientífico para resolver la SSI bajo estudio, poniendo los riesgos al centro de estas dinámicas (Owens, Sadler y Zeidler 2017; Gordillo, 2017).

El metamodelo didáctico propuesto configura así, un marco multidimensional que orienta al profesor de ciencias en la promoción de la competencia en riesgo, a través del esquema de SSI como andamiaje para estructurar experiencias en la clase de ciencias, orientadas al riesgo.

\section{Conclusiones: Pedagogías del Riesgo PARA la ALFABetización Científica}

Ante la COVID-19 que vivimos y los riesgos de que ocurran otras pandemias en el futuro, aunado a las innumerables consecuencias que se prevén próximas dado el cambio climático, urge que la educación científica normalice la presencia de la incertidumbre, el riesgo y los valores en juego como parte intrínseca de la actividad sociocientífica (Owens, Sadler y Zeidler 2017). Necesitamos dejar de evitar y marginar el abordaje de estos aspectos inciertos de la ciencia y encontrar las mejores maneras de trabajarlos didácticamente para conformar una competencia en riesgo, dentro de la alfabetización en ciencias, que prepare al estudiantado hacia el futuro.

No contar con una educación en riesgo puede tener entre sus consecuencias el sobreestimarlo sobrerreaccionar emocional, técnica, económica, políticamente a este- o bien subestimarlo con sus potenciales consecuencias fatales para la vida (Hansen y Hamman 2017).

Las conceptualizaciones teóricas sobre el riesgo han dejado claro su carácter objetivo y técnico, pero al mismo tiempo, su carácter construido y subjetivo. El riesgo se constituye heterogéneamente como un fenómeno que recoge los elementos sociales y materiales, objetivos y subjetivos (Rodríguez 2007) y la educación no puede ignorar esta característica.

Una vez asumida esta constitución heterogénea del riesgo, el siguiente paso es reconocer su presencia ubicua; más que una consecuencia colateral de la actividad científica, el riesgo es un elemento definitorio e inevitable que acompaña a todo proceso de producción y uso de conocimiento en las ciencias. Estos aspectos teóricos del riesgo, a pesar de estar claros teóricamente, no siempre se traducen de esta manera en el contexto de la educación, en donde los riesgos no se asumen todavía como presentes en toda la actividad científica y en donde sigue predominando sólo su faceta objetiva y técnica.

Las propuestas pedagógicas analizadas, contextualizadas en un esquema SSI, como lo propone el metamodelo presentado, tienen el potencial de ofrecer a los estudiantes trabajar las SSI con los dos paradigmas del riesgo; en su faceta técnica, convocando conocimientos científicos específicos, y en su faceta subjetiva, recuperando los significados sociales que este tiene en la SSI bajo análisis.

De las lecciones aprendidas de esta investigación documental podemos aventurarnos a proponer los siguientes objetivos y principios básicos para configurar una pedagogía del riesgo en el aula de ciencias.

1. Objetivo: Desarrollar una competencia en riesgo, asumiéndolo como característica propia y definitoria de la actividad científico-tecnológica; el riesgo es un indicador, en toda práctica científica, de las tensiones y acomodos en las relaciones entre naturaleza-ciencia-tecnología-sociedad (Rodríguez 2007). Una enseñanza de la ciencia sin riesgo no tiene sentido; así como tampoco lo tiene concentrarse solamente en la enseñanza de los aspectos objetivos y técnicos del riesgo, dejando de lado la discusión de su complejidad y de sus aspectos sociales, culturales, políticos, económicos.

2. Principios: Para fomentar la enseñanza del riesgo en la clase de ciencias, estos tres principios pueden resultar útiles para el profesorado (Christensen, 2009):

$i$. Reconocer y lidiar con el riesgo y la incertidumbre como rasgos propios del quehacer científico, que se manifiestan mejor en la medida en que se propician experiencias auténticas y contextualizadas de aprendizaje de las ciencias (Díaz, 2006). 
ii. Explicitar el alcance y las limitaciones que tiene la ciencia en sus contextos, para mostrar que ni sus problemas, ni sus soluciones son neutrales desde el punto de vista ético, social, político, cultural y/o económico

iii. Adecuar la enseñanza de las ciencias de modo tal que la incertidumbre y el riesgo sean analizados desde sus múltiples significados técnicos, culturales y sociales.

A estos tres principios podemos añadir uno más:

$i v$. No basta con enseñar una definición de riesgo, sino que para ser competente en riesgo se requiere aprender a participar del riesgo, esto es, aprender a identificarlo, evaluarlo, gestionarlo y comunicarlo en sus diferentes contextos sociales y culturales.

El riesgo es siempre un fenómeno socialmente percibido, y su enseñanza conlleva comprometerse con una visión más progresista, comprehensiva y compleja de lo que es la alfabetización en ciencias, en la que puedan desplegarse diversas pedagogías del riesgo que contribuyan a que la enseñanza de la ciencias sea más acorde a los tiempos que vivimos, con metodologías activas, humanistas, más transformativas que transmisivas, que logren formar para la acción sociopolítica y para la resolución de preocupaciones reales y problemas globales.

\section{Agradecimientos}

Se agradece el apoyo del Proyecto DGAPA-PAPIIT-IG400920

\section{REFERENCIAS BIBLIOGRÁfICAS}

Beck U. (1992) Risk Society: Towards a New Modernity. London: SAGE.

Bialostok S. (2015) Risk theory and education: Policy and practice. Policy Futures in Education 13 (5), 561-576. ht tps://doi.org/10.1177/1478210315572519

Bialostok S. Whitman R. (2012) Education and the risk society: an introduction. En S. Bialostok, R. Whitman, W. Bradley (Eds), Education and the Risk Society: Theories, Discourse and Risk Identities in Education Contexts (pp. 1-34). Rotterdam: Sense.

Christensen C. (2009) Risk and school science education. Studies in Science Education 45(2), 205-223. https://doi. org/10.1080/03057260903142293

Díaz, F. (2006) Enseñanza situada: vínculo entre la escuela y la vida. México: McGrawHill.

Eijkelhof H. (1986) Dealing with acceptable risk in science education: the case of ionizing radiation. Ethics and social responsibility in science education 2 (189), 189-198. https://doi.org/10.1007/s00411-018-0763-4

FEM. (2019) Reporte de riesgos globales. Foro Económico Mundial. Recuperado de: http://www3.weforum.org/docs /WEF_Global_Risks_Report_2019.pdf

Gordillo M. (2017) El enfoque CTS en la enseñanza de la ciencia y la tecnología. Asunción: Conacyt/OEI.

Hansen J., Hammann M. (2017) Risk in Science Instruction. The Realist and Constructivist Paradigms of Risk. Science \& Education 26, 749-775. https://doi.org/10.1007/s11191-017-9923-1

Howes R. (1975) Radiation risks—a possible teaching topic? Physics Education 10(6), 412-416.

Jasanoff S. (1999) The songlines of risk. Environmental Values 8 (2), 135-152.

Kolstø S. (2006) Patterns in students' argumentation confronted with a risk-focused socio-scientific issue. International Journal of Science Education 28 (14), 1689-1716. https://doi.org/10.1080/09500690600560878

Lee H., Yoo J., Choi K., Kim S., Krajcik J., Herman B., Zeidler D. (2013) Socioscientific Issues as a Vehicle for Promoting Character and Values for Global Citizens, International Journal of Science Education 35 (12) 2079-2113. https://doi.org/10.1080/09500693.2012.749546

Levinson R. (2006) Towards a Theoretical Framework for Teaching Controversial Socio-scientific Issues. International Journal of Science Education 28 (10) 1201-1224. https://doi.org/10.1080/09500690600560753 
Mack, O., Khare, A. (2016) Perspectives on a VUCA world. En O. Mack et al. (eds.) Managing in a VUCA world (pp. 3-19), Switzerland: Springer.

Martínez P., Díez F. (2020, 15 de abril) La crisis del coronavirus afecta a 1570 millones de estudiantes icómo encarar el tsumani? The Conversation. Recuperado de: https://theconversation.com/amp/la-crisis-del-coronavirus-afec ta-a-1-570-millones-de-estudiantes-como-encarar-el-tsunami-136408

Millar R. (2006) Twenty first Century science: insights from the design and implementation of a scientific literacy approach in school science. International Journal of Science Education 28(13), 1499-1521. https://doi.org/10. 1080/09500690600718344

Nikiforidou Z. (2017) Risk literacy: Concepts and pedagogical implications for early childhood education. Contemporary Issues in Early Childhood 18 (3), 322-332. https://doi.org/10.1177/1463949117731027

Owens D., Sadler T., Zeidler D. (2017) Controversial issues in the science classroom. Phi Delta Kappan 99 (4), 4549. https://doi.org/10.1177/0031721717745544

Pedretti E., Nazir Y. (2011) Currents in STSE education: Mapping a complex field, Science Education 95(4), 601-626. https://doi.org/10.1002/sce.20435

Pedretti E., Nazir Y. (2016) Science, Technology and Society. En R. Gunstone (Ed.), Encyclopedia of Science Education (pp. 932- 935), Dordrecht: Springer.

Ravetz J. (1997) Simple scientific truths and uncertain policy realities: implications for science education. Studies in Science Education, 30(1), 5-18. https://doi.org/10.1080/03057269708560101

Renn O. (1992) Concepts of risk: A classification. En S. Krimsky, D. Golding (Eds.) Social theories of risk (pp. 5382). Westport: Praeger.

Riechard D. (1993) Risk literacy: Is it the missing link in environmental education? The Journal of Environmental Education 25(1), 8-12.

Rodríguez H. (2007) Dinámicas de constitución del riesgo: una propuesta composicionalpara gobernar el riesgo. Donostia: Universidad del País Vasco.

Ryder J. (2001) Identifying science understanding for functional scientific literacy. Studies in Science Education 36 (1), 1-44. https://doi.org/10.1080/03057260108560166

Sadler T. (2011) Socio-scientific Issues-Based Education: What We Know About Science Education in the Context of SSI. En T. Sadler (Ed.), Socio-scientific Issues in the Classroom (pp 355-369). Dordrecht: Springer.

Sadler T., Dawson V. (2012) Socio-scientific Issues in Science Education: Contexts for the Promotion of Key Learning Outcomes. En B.J. Fraser (Eds.), Second International Handbook of Science Education (pp. 799- 809). Dordrecht: Springer.

Sadler T., Donnelly, L. (2006) Socioscientific Argumentation: The effects of content knowledge and morality, International Journal of Science Education 28(12), 1463-1488. https://doi.org/10.1080/09500690600708717

Schenk L., Hamza K., Enghag M., Lundegård I., Arvanitis L., Haglund K., Wojcik A. (2019) Teaching and discussing about risk: seven elements of potential significance for science education, International Journal of Science Education 41 (9), 1271-1286. https://doi.org/10.1080/09500693.2019.1606961

Zeidler D, Herman B., Sadler T. (2019) New directions in socioscientific issues research. Disciplinary and Interdisciplinary Science Education Research 1, 1-11 https://doi.org/10.1186/s43031-019-0008-7

Zeidler D. (2016) Socioscientific Issues. En R. Gunstone (Ed.), Encyclopedia of Science Education (pp. 998-1002). Dordrecht: Springer.

Zeidler D., Nichols B. (2009) Socioscientific Issues: Theory and Practice. Journal of Elementary Science Education, 21(2), 49- 58. https://doi.org/10.1007/BF03173684

Zeidler D., Sadler T. (2011) An inclusive view of scientific literacy: core issues and future directions of socioscientific reasoning. En: C. Linder, L Ostman, D. Roberts, P. Wickman, G. Erickson, A MacKinnon (Eds.) Promoting scientific literacy: science education research in transaction (pp 176-192). New York: Routledge. 
Liliana Valladares. Pedagogías del Riesgo: alfabetización científica en tiempos de Pandemia

\section{INFORMACIÓN ADICIONAL}

Para citar este articulo: Valladares, L. (2022) Pedagogías del Riesgo: alfabetización científica en tiempos de pandemia. Revista Eureka sobre Enseñanza y Divulgación de las Ciencias 19(1), 1301. doi: 10.25267/ Rev_Eureka_ensen_divulg_cienc.2022.v19.i1.1301 